Revista de Psicología de la PUCP. Vol. XXII, 12004

\title{
Comparación de la agresión infantil en dos grupos culturales
}

\author{
María Luisa Roa ${ }^{1}$, María Victoria Del Barrio² y \\ Miguel Ángel Carrasco ${ }^{3}$ \\ Universidad Nacional de Educación a Distancia
}

En este trabajo se estudian distintas emociones y comportamientos: Inestabilidad Emocional, Agresividad Física y Verbal, y Conducta Prosocial. En primer lugar, se analizan las correlaciones entre las puntuaciones pertenecientes a las distintas fuentes informantes (jóvenes y profesores). Se comparan también las diferencias entre distintas culturas (española y peruana). La muestra está compuesta por 50 niños y adolescentes; 25 peruanos y 25 españoles. Los datos muestran que, en Agresividad, no hay diferencias significativas entre las puntuaciones de los jóvenes y los maestros. Sin embargo, aparece una mayor correlación entre jóvenes y profesores peruanos que entre jóvenes y profesores españoles. Por otro lado, los adolescentes peruanos tienen niveles más elevados en Inestabilidad Emocional, mientras que los españoles alcanzan puntuaciones medias más altas en Conducta Prosocial y Agresividad. Estas diferencias entre los grupos pueden estar motivadas por factores culturales.

Palabras clave: agresividad, conducta prosocial, inestabilidad emocional, niños, adolescentes, concordancia entre fuentes.

\section{Comparison of infantile aggression in two different cultural groups}

Different emotions and behaviors were examined in this study: Emotional Instability, Physical and Verbal Aggression, and Prosocial Behavior. First of all, we analyzed the correlation between ratings from different sources (children or teachers). Cultural differences were also discussed with regards to a sample of 50 children and adolescents, 25 Peruvians and 25 Spanish. Data showed non-significant differences in Aggressiveness between children and teachers' ratings. Nevertheless, the correlation is higher between Peruvian children and teachers than between Spanish ones. On the other hand, Peruvian teenagers showed higher levels of Emotional Instability whereas the Spanish got higher average ratings in Prosocial Behavior and Aggressiveness. These data could be due to cultural differences.

Key words: aggressiveness, emotional instability, prosocial behavior, children, concordance.

1 Doctora en Psicología. Supervisora del Servicio de Psicología aplicada de la UNED. Especialista en problemas infantiles y hábitos de crianza, habiendo publicado diferentes artículos y trabajos sobre este tema.

2 Doctora en Psicología. Profesora títular de la Facultad de Psicología de la UNED. Ha publicado libros y artículos sobre el tema de problemas infantiles. Correo electrónico: vbarrio@psi.uned.es

3 Doctor en Psicología. Profesor Asociado de la Facultad de Psicología de la UNED. Especialista en problemas infantiles. Tiene numerosos artículos sobre agresión, depresión y otras emociones infantiles. 

En los últimos años los medios de comunicación, radio, televisión y periódicos, informan cada día sobre la escalada de violencia que se vive en las escuelas y también en las familias. Los niños son más violentos, por tanto, se ha de analizar por qué se produce este fenómeno y que relación tiene con los contextos sociales en donde estas conductas se dan.

El National Center for Juvenile Justice de Estados Unidos estima que en el año 2010 la violencia juvenil entre los 10-17 años crecerá $58 \%$ en robos, $66 \%$ en violaciones, $129 \%$ en asaltos y $142 \%$ en muertes. Estos son datos de uno de los países en donde el bienestar ha alcanzado niveles más altos, pero también donde los índices de criminalidad común siempre han sido más elevados.

La conducta violenta debe ser entendida desde un punto de vista interactivo que tenga en cuenta los distintos elementos que pueden estar implicados en su explicación. La conducta en general, y la violenta en especial, se compone de ingredientes sociales, personales y orgánicos, integrados de una manera intrincada y de difícil análisis. Berkowitz (1993) sostiene que los elementos que intervienen en la gestación de la conducta violenta son muy plurales e interactúan entre sí de manera muy compleja. Entre estos elementos están: el tipo de sistema nervioso, disfunciones neurofisiológicas, lesiones cerebrales, problemas familiares (desestructuración familiar, malas relaciones, abandono, abuso, violencia doméstica), tipo de temperamento y personalidad, impulsividad, acceso a armas, conducta disruptiva en la infancia, grupos de compañeros, gratificación de la violencia, permisividad social y otros muchos. De todos ellos unos son de naturaleza personal y otros de carácter más social. 
Tremblay (2003) sostiene que los niños comienzan su conducta agresiva muy precozmente. Entre el primer y segundo año se produce el pico más alto de conductas agresivas, aunque éstas tengan poca intensidad dada la edad de los sujetos. A medida que el niño crece desciende el número de conductas agresivas gracias a los sistemas inhibitorios que produce el mundo circundante y el autocontrol. Por tanto, es importante analizar tanto el potencial de agresión de cada persona como los sistemas inhibitorios que se le brinda. Sólo unas pocas personas permanecen agresivos a lo largo de toda la vida. Hay evidencia de que el comportamiento del niño en la escuela es especialmente relevante para predecir la probabilidad de la permanencia del niño en la conducta agresiva hasta la edad adulta. Existe una fuerte correlación entre conductas delictivas en la pubertad y las puntuaciones de los maestros en conducta agresiva en la escuela. Por ello, es especialmente importante la evaluación que hacen los maestros a este respecto.

Considerando estos antecedentes, en este estudio se analizan algunas de las características personales que potencian la conducta violenta de los individuos jóvenes, y que se podrían englobar en lo que Berkowitz (1993) llamaba disposición previa. En este caso, nos centramos en la característica de Inestabilidad Emocional. Desde el punto de vista de las influencias ambientales se estudia la Conducta Prosocial, puesto que es aprendida y variable en distintas culturas. También se estudia la conducta agresiva como un conjunto de acciones que pueden o no presentarse en determinados sujetos. Por último, se intenta conocer qué diferencias se dan en cada una de estas variables en dos culturas muy diferentes: la de jóvenes españoles urbanos y la de jóvenes peruanos ubicados en una zona selvática.

Las personas con altos niveles de Inestabilidad Emocional comienzan a manifestar desde edades más tempranas una tendencia a experimentar ansiedad, depresión, cambios bruscos de humor, irritabilidad, elevada reactividad a acontecimientos aversivos, problemas 
de sueño y otros problemas psicosomáticos. En relación con la agresividad, la inestabilidad emocional o el neuroticismo, se ha asociado con un aumento de conductas agresivas (Bandura, Barbaranelli, Caprara \& Pastorelli, 1996; Caprara, Barbaranelli \& Comrey, 1992; Caprara, Barbaranelli \& Zimbardo, 1996; Caprara, Manzi \& Perugni, 1992; Caprara \& Pastorelli, 1989; Caprara \& Zimbardo, 1996; Heaven, 1996). Similarmente, la Conducta Prosocial, entendida como un conjunto de manifestaciones de ayuda y altruismo que una persona pone en marcha cuando interactúa con los otros, es un importante inhibidor de la conducta agresiva (Pérez Delgado \& Mestre, 1999).

\section{Metodología}

\section{Participantes}

La muestra general está constituida por 50 participantes, 38 varones ( $76 \%$ ) y 12 mujeres (24\%), 25 de nacionalidad española y 25 de nacionalidad peruana. La edad media de los participantes es 14,5 años, con 2,4 de desviación estándar. La descripción por sexo y edad de cada uno de los grupos puede verse en el Cuadro 1.

El grupo de jóvenes peruanos procede de la selva amazónica (Santa Rita de Castilla) y pertenecen a un colegio público de ese lugar. El grupo de jóvenes españoles proviene de una amplia muestra de distintos colegios públicos de Madrid. Ambos grupos se seleccionaron al azar.

\section{Instrumentos}

- Escala de Agresividad Física y Verbal (AFV) (Caprara \& Pastore1li, 1993): compuesta por 20 elementos. Cinco de ellos son considerados elementos de control, por lo que no son computables 
para la puntuación total. La respuesta a cada ítem se realiza en una escala de 1 a 3 puntos ( 1 nunca; 2 algunas veces; y, 3 a menudo) que indica la frecuencia de la conducta evaluada.

- Escala de Inestabilidad Emocional (IE) (Caprara \& Pastorelli, 1993): consta de 20 elementos, 5 de los cuales no puntúan. La respuesta a cada ítem se efectúa en una escala de 3 puntos que va desde nunca hasta a menudo.

- Escala de Conducta Prosocial (CP) (Caprara \& Pastorelli, 1993): consta de 20 elementos de los cuales, al igual que las anteriores, cinco no puntúan. La respuesta a cada elemento, aquí también, se efectúa en una escala de 3 puntos.

Para este estudio se ha utilizado la adaptación española de las pruebas realizada por Del Barrio, Moreno y López (2001).

\section{Procedimiento}

Se obtuvo los datos mediante dos fuentes diferentes, el joven y el maestro. El primer paso fue pedir autorización a los responsables de los centros educativos para evaluar a los participantes. Una vez concedido el permiso, se fijó día y hora para pasar las pruebas. A los jóvenes peruanos, procedentes de un colegio público de Santa Rita de Castilla, se administraron las escalas de AFV, IE y CP en una misma sesión. Previamente habían recibido instrucciones del evaluador sobre la forma de completarlos. El tiempo aproximado que duró la sesión fue de media hora. Todos los jóvenes prestaron su colaboración de forma voluntaria y desinteresada.

El grupo de jóvenes españoles fue evaluado en idénticas condiciones que la muestra peruana. Los instrumentos se completaron dando las mismas instrucciones y en el mismo orden. A los profesores, uno por cada muestra, se les dejaron las pruebas para que una vez 
completadas fueran devueltas, recomendándoles que lo hicieran con la mayor brevedad posible.

Una vez obtenidos los datos, se procedió a su corrección y análisis. Los resultados estadísticos se obtuvieron con el programa SPSS.10.0.

\section{Resultados}

La descripción de la muestra según sexo, edad y lugar de procedencia, queda recogida en el Cuadro 1 . Como se ve, los sujetos procedentes de ambos grupos son equiparables en cuanto al número y sexo; sin embargo, la edad del grupo peruano es más alta. Esto se debe a la distinta distribución de la edad en ambos grupos en los mismos niveles.

\section{Cuadro 1}

Descripción de la muestra por sexo y edad en cada uno de los grupos estudiados

\begin{tabular}{ccc}
\hline & $\begin{array}{c}\text { Grupo } \\
\text { peruano }\end{array}$ & $\begin{array}{c}\text { Grupo } \\
\text { español }\end{array}$ \\
\hline Varón & 19 & 19 \\
Mujer & 6 & 6 \\
Total & 25 & 25 \\
\hline Edad $M$ & 16.52 & 12.44 \\
$(D E)$ & $(1,50)$ & $(0.96)$ \\
\hline
\end{tabular}

Las puntuaciones medias y desviaciones estándar de las escalas evaluadas en cada uno de los grupos se recogen en el Cuadro 2. En dicho cuadro aparecen los descriptivos mencionados según la fuente que informa. 


\section{Cuadro 2}

Media y desviación estándar según la fuente que informa en cada uno de los grupos culturales

\begin{tabular}{|c|c|c|c|c|c|c|c|}
\hline & & \multicolumn{2}{|c|}{ Españoles } & \multicolumn{2}{|c|}{ Peruanos } & \multicolumn{2}{|c|}{ Total } \\
\hline & & Joven & Profesor & Joven & Profesor & Joven & Profesor \\
\hline $\begin{array}{l}\text { Inestabilidad } \\
\text { Emocional }\end{array}$ & $\begin{array}{c}M \\
D E\end{array}$ & $\begin{array}{l}24,28 \\
(4,51)\end{array}$ & $\begin{array}{l}24,08 \\
(6,64) \\
\end{array}$ & $\begin{array}{l}25,72 \\
(3,68)\end{array}$ & $\begin{array}{l}25,00 \\
(5,02)\end{array}$ & $\begin{array}{l}25,00 \\
(4,14)\end{array}$ & $\begin{array}{l}24,67 \\
(5,83)\end{array}$ \\
\hline $\begin{array}{l}\text { Conducta } \\
\text { Prosocial } \\
\end{array}$ & $\begin{array}{c}M \\
D E \\
\end{array}$ & $\begin{array}{l}26,16 \\
(2,65) \\
\end{array}$ & $\begin{array}{l}23,64 \\
(4,46) \\
\end{array}$ & $\begin{array}{l}23,76 \\
(2,47) \\
\end{array}$ & $\begin{array}{l}24,12 \\
(5,56) \\
\end{array}$ & $\begin{array}{l}24,92 \\
(2,78) \\
\end{array}$ & $\begin{array}{l}23,88 \\
(5,00) \\
\end{array}$ \\
\hline Agresividad & $\begin{array}{c}M \\
D E\end{array}$ & $\begin{array}{l}22,17 \\
(4,87)\end{array}$ & $\begin{array}{l}23,16 \\
(6,75)\end{array}$ & $\begin{array}{l}21,12 \\
(4,40)\end{array}$ & $\begin{array}{l}21,28 \\
(4,56)\end{array}$ & $\begin{array}{l}21,70 \\
(4,69)\end{array}$ & $\begin{array}{l}22,22 \\
(5,78)\end{array}$ \\
\hline
\end{tabular}

Diferencias en las medidas de Inestabilidad Emocional, Conducta Prosocial y Agresividad según las fuentes informantes

Se realizaron distintas comparaciones no paramétricas entre las medidas según la fuente informante. Los resultados no pusieron de manifiesto diferencias significativas entre fuentes, ni en el total de la muestra ni en cada uno de los grupos procedentes de las nacionalidades estudiadas.

A pesar de que las diferencias no fueron significativas, se puede destacar que las puntuaciones medias en Conducta Prosocial y Agresividad de los jóvenes españoles son más elevadas que las de los jóvenes peruanos, mientras que los jóvenes peruanos tienen puntuaciones medias superiores en Inestabilidad Emocional. Es decir, los jóvenes españoles muestran mayores conductas de ayuda tales como "ser amable o ayudar a los demás", y también las relacionadas con hacer daño a los otros como "pelearse y decir mentiras". En los jóvenes peruanos, las conductas más elevadas se refieren al estado emocional, por ejemplo, "ser impaciente, enfadarse o estar de mal humor". 
Concordancia en las medidas de Inestabilidad Emocional, Conducta Prosocial y Agresividad según las fuentes informantes

Para hallar la concordancia entre las distintas fuentes se analizaron las correlaciones entre las medidas obtenidas del propio joven y las brindadas por el profesor en cada una de las variables. Como puede verse en los Cuadros 3, 4 y 5 se calcularon las correlaciones parciales controlando la edad en el conjunto de la muestra y en cada uno de los grupos por separado, en el grupo de jóvenes españoles y en el de jóvenes peruanos.

En el total de la muestra las correlaciones que resultaron significativas entre las distintas fuentes fueron dos: la primera entre Agresividad autoinformada y Agresividad heteroinformada, y la segunda entre Agresividad heteroinformada e Inestabilidad Emocional autoinformada. Por tanto, la concordancia entre fuentes es significativamente convergente en la variable Agresividad. La inestabilidad autopercibida por el joven (conducta interiorizada) es, sin embargo, percibida por el maestro, lo que indica que esa conducta del joven tiene signos observables.

\section{Cuadro 3}

Correlaciones Parciales entre maestros y alumnos controlando la edad en cada una de las medidas de Inestabilidad Emocional, Conducta Prosocial y Agresividad en el total de la muestra $(n=50)$

\begin{tabular}{lccc}
\hline & $\begin{array}{c}\text { Inest. Emocional } \\
\text { autoinformada }\end{array}$ & $\begin{array}{c}\text { Cond. Prosocial } \\
\text { autoinformada }\end{array}$ & $\begin{array}{c}\text { Agresividad } \\
\text { autoinformada }\end{array}$ \\
\hline $\begin{array}{l}\text { Inest. Emocional } \\
\text { heteroinformada }\end{array}$ & 0,24 & 0,09 & 0,20 \\
\hline $\begin{array}{l}\text { Cond. Prosocial } \\
\text { heteroinformada }\end{array}$ & $-0,009$ & 0,23 & $-0,12$ \\
\hline $\begin{array}{l}\text { Agresividad } \\
\text { heteroinformada }\end{array}$ & $-0,28 *$ & 0,05 & $0,42 * *$ \\
\hline$* 0,05 ; * * p<0,01$ & &
\end{tabular}


Si consideramos la concordancia en cada uno de los grupos por separado, en el de jóvenes peruanos la coincidencia entre la información obtenida por los profesores y los jóvenes es distinta que en el total de la muestra. Cuando analizamos la muestra peruana, las correlaciones entre profesor y alumno son más altas y significativas en las variables de Agresividad y de Inestabilidad Emocional aunque sigue el mismo patrón que la muestra total. Esta coincidencia se ha puesto de manifiesto tanto en la evaluación de conductas claramente exteriorizadas, es decir, conductas que manifiestan una exteriorización de las emociones (por ejemplo, la Agresividad) como en conductas más interiorizadas (Inestabilidad Emocional). Asimismo, son destacables también las correlaciones entre distintas variables tales como la Agresividad autoinformada (es decir, la evaluada a través de la información que da el propio joven) y la Inestabilidad Emocional evaluada por el profesor (Cuadro 4).

\section{Cuadro 4}

Correlaciones parciales entre maestros y alumnos controlando la edad en cada una de las medidas de Inestabilidad Emocional, Conducta Prosocial y Agresividad en el grupo de peruanos $(n=25)$

\begin{tabular}{lccc}
\hline & $\begin{array}{c}\text { Inest. Emocional } \\
\text { autoinformada }\end{array}$ & $\begin{array}{c}\text { Cond. Prosocial } \\
\text { autoinformada }\end{array}$ & $\begin{array}{c}\text { Agresividad } \\
\text { autoinformada }\end{array}$ \\
\hline $\begin{array}{l}\text { Inest. Emocional } \\
\text { Heteroinformada }\end{array}$ & $0,42^{*}$ & $-0,01$ & $0,44^{*}$ \\
\hline $\begin{array}{l}\text { Cond. Prosocial } \\
\text { Heteroinformada }\end{array}$ & $-0,01$ & 0,15 & $-0,09$ \\
\hline $\begin{array}{l}\text { Agresividad } \\
\text { heteroinformada }\end{array}$ & $0,49^{*}$ & 0,12 & $0,65^{* *}$ \\
\hline
\end{tabular}

${ }^{*} p<0,05 ; * * p<0,01$

Comparando las correlaciones entre fuentes en ambos grupos parece que el conocimiento que el profesor del grupo peruano tiene de sus alumnos es más empático que el del profesor del grupo español. 
Comparación de la agresión infantil en dos grupos culturales

En el caso de la muestra española, ninguna correlación entre fuentes resultó ser significativa (Cuadro 5).

\section{Cuadro 5}

Correlaciones parciales entre fuentes controlando la edad en cada una de las medidas de Inestabilidad Emocional, Conducta Prosocial y Agresividad en el grupo de españoles $(n=25)$

\begin{tabular}{lccc}
\hline & $\begin{array}{c}\text { Inest. Emocional } \\
\text { autoinformada }\end{array}$ & $\begin{array}{c}\text { Cond. Prosocial } \\
\text { autoinformada }\end{array}$ & $\begin{array}{c}\text { Agresividad } \\
\text { autoinformada }\end{array}$ \\
\hline $\begin{array}{l}\text { Inest. Emocional } \\
\text { heteroinformada }\end{array}$ & 0,16 & 0,18 & 0,04 \\
\hline $\begin{array}{l}\text { Cond. Prosocial } \\
\text { heteroinformada }\end{array}$ & $-0,11$ & 0,31 & $-0,20$ \\
\hline $\begin{array}{l}\text { Agresividad } \\
\text { heteroinformada }\end{array}$ & 0,17 & $-0,04$ & 0,31 \\
\hline
\end{tabular}

${ }^{*} p<0,05 ; * * p<0,01$

\section{Discusión}

Si comparamos las puntuaciones medias obtenidas en este estudio con el realizado por Caprara y Pastorelli (1993) en una muestra de niños italianos utilizando los mismos instrumentos, observamos que los italianos puntúan más alto en las escalas de Inestabilidad Emocional $(28,73)$ y Agresividad $(24,33)$ que los españoles y peruanos. En la escala de Conducta Prosocial, los italianos tienen puntuaciones semejantes a la de los peruanos $(23,80)$, pero inferiores a las de los españoles. Por otro lado, en el estudio realizado por Del Barrio, Moreno y López (2001) con una muestra de niños españoles y con los mismos instrumentos, las puntuaciones en Agresividad $(21,63)$ e Inestabilidad Emocional $(24,09)$ son semejantes a las halladas en las dos nacionalidades consideradas para este estudio. Las correlaciones entre profesores y alumnos en este estudio son muy semejantes a las 
encontradas por Caprara y Pastorelli (1993) en las mismas escalas (EI, 0,25; CP, 0,21 y AFV, 0,28) y con niños italianos.

La relación encontrada entre las variables Inestabilidad Emocional y Agresividad en la muestra de peruanos se ha mostrado similar a la hallada por Verdugo-Arstark y Fordham (2002) en una muestra anglosajona.

En cuanto a los resultados obtenidos sobre la concordancia entre fuentes, se obtuvo diferencias en función del grupo cultural y del tipo de variables evaluadas. En general, tomando el total de la muestra, la concordancia entre fuentes es más potente en la variable de Agresividad, pero resultó ser mucho menor en el resto (Conducta Prosocial e Inestabilidad Emocional). No obstante, cuando la concordancia se estudia en cada uno de los grupos culturales (por un lado el grupo peruano y por el otro el de españoles), la convergencia entre informadores es superior en el grupo de peruanos.

La literatura sobre este tema ha informado en numerosas ocasiones sobre la baja concordancia entre fuentes en la evaluación infantil de problemas emocionales (Del Barrio \& Moreno, 1996; Jané, Araneda, Valero \& Doménech, 2000; Kashani, Holcomb \& Orvaschel, 1986; Martini, Strayhorn \& Puig-Antich, 1990), y sobre cómo la concordancia se incrementa cuando se trata de problemas más exteriorizados, como es el caso de la Agresividad. Las conductas exteriorizadas, caracterizadas por una expresión externa y, por tanto, por una mayor visibilidad y accesibilidad, posibilitan una mejor convergencia entre distintos evaluadores que las conductas más interiorizadas y privadas (Caprara \& Pastorelli, 1993; Del Barrio et al., 2001; Epkins, 1993; Pastorelli et al., 1997).

Las diferencias de concordancia entre distintas culturas no han sido tan frecuentemente exploradas en la literatura, por lo que resulta difícil hacer comparaciones. En el presente trabajo los resultados 
indican que el grupo de peruanos posee una mayor convergencia de fuentes, tanto en las conductas exteriorizadas como en las interiorizadas. La razón de este resultado puede basarse en las posibles diferencias culturales y contextuales manifiestas en la muestra peruana, como son el tipo de relación que se establece entre profesor y alumno y el menor número de alumnos que un profesor tiene a su cargo, lo que quizás posibilite un tipo de comunicación más cercana e intensa y un mayor conocimiento del alumno. Los niños españoles proceden de aulas más numerosas que los niños peruanos. Este dato puede ser la explicación última de las diferencias encontradas en la concordancia más alta entre profesor y alumno en la muestra del Perú.

Las limitaciones de este estudio no permiten concluir definitivamente sobre estos resultados (tamaño reducido de la muestra), debería ser replicado en posteriores estudios que permitan el uso de pruebas psicométricas más potentes y con mayor generalidad de los datos. A pesar de las limitaciones, los resultados son muy similares a los de otros trabajos y están en consonancia a los obtenidos en muestras más amplias. Por todo ello, se requeriría profundizar en el estudio e investigación de estos problemas y seguir indagando en la dirección de lo aquí apuntado.

\section{Referencias}

Bandura, A., Barbaranelli, C., Caprara, G. \& Pastorelli, C. (1996). Multifaceted impact of self-efficacy beliefs of academic functioning. Child Development, 67, 1206-1222.

Berkowitz, L. (1993). Aggression: Its causes, consequences, and control. Filadelfia: McGraw Hill.

Caprara, G. V., Barbaranelli, C. \& Zimbardo, P. (1996). Understanding the complexity of human aggression: Affective, cognitive, and social dimensions of individual differences in propensity 
toward aggression. European Journal of Personality, 10, 133155.

Caprara, G. V., Barbaranelli, C. \& Comrey, A. L. (1992). A personological approach to the study of aggression. Personality and Individual Differences, 13, 77-84.

Caprara, G. V., Manzi, J. \& Perugini, M. (1992). Investigating guilt in relation to emotionality and aggression. Personality and Individual Differences, 13, 519-532.

Caprara, G. V. \& Pastorelli, C. (1989). Toward a reorientation of research on aggression. European Journal of Personality, 3, 121138.

Caprara, G. V. \& Zimbardo, P. G. (1996). Aggregation and amplification of marginal deviations in the social construction of personality and maladjustment. European Journal of Personality, 10, 79-110.

Del Barrio, V. \& Moreno, C. (1996). Evaluación de la depresión y la ansiedad infantil. Ansiedad y Estrés, 2, 131-158.

Del Barrio, V., Moreno, C. \& López, R. (2001). Evaluación de la agresión y la inestabilidad emocional con niños españoles: su relación con la depresión. Clínica y Salud, 12(1), 33-50.

Epkins, C. (1993). A preliminary comparison of teacher ratings and child self-report of depression, anxiety, and aggression in inpatient and elementary school samples. Journal of Abnormal Child Psychology, 21, 649-661.

Heaven, P. (1993). Personality predictors of self-reported delinquency. Personality and Individual Differences, 13, 67-79.

Jané, M., Araneda, N., Valero, S. \& Doménech, E. (2000). Evaluación de la sintomatología depresiva del preescolar: correspondencia entre los informes de padres y maestros. Psicotema, 12, 212-215.

Kashani, J. H., Holcomb, W. R. \& Orvaschel, H. (1986). Depression and depressive symptoms in preschool children from the general population. American Journal of Psychiatry, 143(9), 1138-1143. Martini, D. R., Strayhorn, J. M. \& Puig-Antich, M. D. (1990). A 
symptom self-report measure for preschool children. American Academy of Child and Adolescent Psychiatry, 29, 594-600.

Pérez Delgado, E. \& Mestre, V. (1999). Psicología moral y crecimiento personal. Barcelona: Ariel.

Pastorelli, C., Barbaranelli, C., Cermak, L., Rozsa, S. \& Caprara, G. V. (1997). Measuring emotional instability, prosocial behavior and aggression in pre-adolescents: A cross-national study. Personality and Individual Differences, 23, 691-703.

Tremblay, R. (2003). Los orígenes de la violencia de los jóvenes. Acción Psicológica, 1(2), 63-72.

Verdugo-Arstark, H. \& Fordham, U. (2002). The relationship of negative emotionality, hostility attributional bias, and social orientation to overt and relational aggression. Dissertation Abstracts International, 63(1), 563. 